\title{
Global Aerodynamic Modeling for Stall/Upset Recovery Training Using Efficient Piloted Flight Test Techniques
}

\author{
Eugene A. Morelli ${ }^{1}$ \\ NASA Langley Research Center, Hampton, Virginia, 23681 \\ Kevin Cunningham ${ }^{2}$ \\ NASA Langley Research Center, Hampton, Virginia, 23681 \\ Melissa A. Hill ${ }^{3}$ \\ Unisys Corporation, NASA Langley Research Center, Hampton, Virginia, 23681
}

\begin{abstract}
Flight test and modeling techniques were developed for efficiently identifying global aerodynamic models that can be used to accurately simulate stall, upset, and recovery on large transport airplanes. The techniques were developed and validated in a high-fidelity fixed-base flight simulator using a wind-tunnel aerodynamic database, realistic sensor characteristics, and a realistic flight deck representative of a large transport aircraft. Results demonstrated that aerodynamic models for stall, upset, and recovery can be identified rapidly and accurately using relatively simple piloted flight test maneuvers. Stall maneuver predictions and comparisons of identified aerodynamic models with data from the underlying simulation aerodynamic database were used to validate the techniques.
\end{abstract}

\section{Nomenclature}

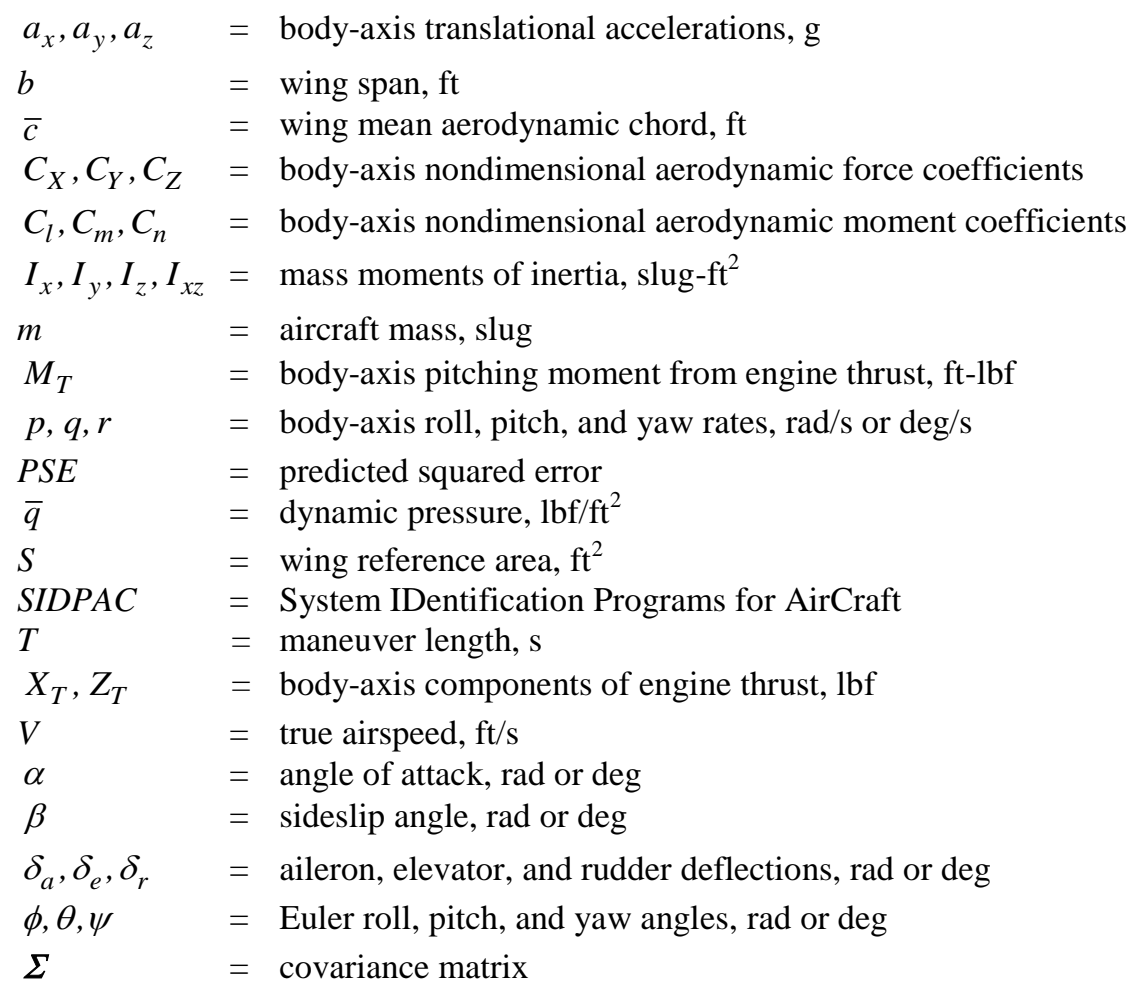

\footnotetext{
${ }^{1}$ Research Engineer, Dynamic Systems and Control Branch, MS 308, AIAA Associate Fellow

${ }^{2}$ Research Engineer, Flight Dynamics Branch, MS 308, AIAA Senior Member

${ }^{3}$ Software Engineer, Simulation Development and Analysis Branch, MS 129
} 


$\begin{array}{lll}\frac{\text { superscripts }}{T} & \\ T & =\text { transpose } \\ \cdot & =\text { time derivative } \\ -1 & =\text { matrix inverse } \\ \text { subscripts } & \\ c g & =\text { center of gravity } \\ o & =\text { reference value or base term }\end{array}$

\section{Introduction}

$\mathrm{O}_{0}^{\mathrm{N}}$ N August 1, 2010, the Airline Safety and Federal Aviation Administration Extension Act of 2010 (H.R. 5900) became law in the United States. This law was intended to improve aviation safety by addressing problems that could have played a role in the February 2009 crash of Colgan Air Flight 3407. One of the provisions in the law directs the Federal Aviation Administration (FAA) to ensure that commercial airline pilots are trained to recover from stalls and upsets, where an upset is generally defined as pitch attitude outside the range $[-10,25]$ degrees, or roll attitude outside the range $[-45,45]$ degrees, or airspeed inappropriate for the conditions ${ }^{1,2}$. Accurate flight simulation for aircraft stall, upset, and recovery is required to enable the effective pilot training necessary to comply with the law, and thereby improve aviation safety.

Typically, flight simulations are based on aerodynamic data collected from wind tunnel tests or generated by computational methods such as computational fluid dynamics (CFD). For large transport aircraft, the aerodynamic database used for flight simulation often does not include data required for accurate stall upset and recovery training ${ }^{1,2}$. Although current flight simulators rely on flight data for validation, flight validation is limited for stall upset conditions. The situation has been widely recognized in government and industry, and is being addressed with research that expands wind-tunnel testing and CFD to include much larger ranges of motion and control variables ${ }^{3-8}$. With expanded flight envelope data, flight simulations can be built to more accurately represent aircraft flight characteristics in stall upset and recovery conditions.

The present work examines an alternate approach for obtaining an accurate aerodynamic model over extended flight envelopes. The approach involves efficient piloted flight testing and nonlinear aerodynamic modeling that can produce accurate global aerodynamic models based on flight data alone. The advantages of this approach are time and cost savings, in addition to better fidelity that can be expected from generating global aerodynamic models using direct measurements on the real full-scale airplane in flight, as opposed to using subscale wind-tunnel models or mathematical representations of the aircraft and the air flow. The latter approaches have accuracy limitations because of effects such as model scale and geometry differences compared to the full-scale aircraft, wind tunnel wall and sting interference, wind tunnel flow angularity, Reynolds number differences, turbulence modeling deficiencies, grid geometry deficiencies for the aircraft and flow field, and high costs. Using flight test methods to generate the aerodynamic model directly avoids all of these problems, although the typical problems associated with any flight test still remain, e.g., sensor data quality, executing flight test maneuvers with sufficient data information content, high cost, safety risk, and practical considerations. The methods described in this work are intended to be a practical, cost-effective, and efficient means to improve flight simulation in stall, upset, and recovery flight conditions, to help comply with the law requiring pilot training in these conditions for improved aviation safety. Furthermore, the FAA requires flight data matching criteria to be satisfied as part of the certification process for commercial flight training simulators. If the aerodynamic model for flight simulation is generated accurately from flight data directly, this requirement could be satisfied without further work.

Previous work in research flight test environments studied effective flight test maneuvers ${ }^{9-12}$ and nonlinear modeling techniques ${ }^{11,12}$ for efficiently generating global aerodynamic models from flight test data alone. The present work refines and extends those techniques, and applies them to the practical problem of generating high-fidelity flight simulation for large transport airplanes over an expanded flight envelope. The work was done in a flight simulation environment, so that the effectiveness of the modeling techniques could be evaluated by directly comparing the underlying aerodynamic database used to generate the simulated flight data with the aerodynamic models identified from the simulated flight data alone.

The next section describes the simulation facility used for this work. Following that is a description of the piloted flight test maneuver technique, and some evaluation and discussion of the resulting simulated flight data. Section IV describes the global aerodynamic modeling technique, which combines multivariate polynomials, polynomial splines, orthogonal function modeling theory, and a statistical modeling metric to autonomously identify 
compact global aerodynamic models for nondimensional force and moment coefficients. The piloted flight test maneuvers and global aerodynamic modeling method are then demonstrated using simulated flight data with realistic aerodynamic nonlinearities and stall characteristics. Direct comparisons of the underlying aerodynamic database and the identified global aerodynamic models are shown in Section V. Prediction results are presented in Section VI.

All of the experiment design, data analysis, and modeling tasks included in this work were done using system identification software written in MATLAB ${ }^{\circledR}$, called System IDentification Programs for AirCraft, or SIDPAC ${ }^{13}$. SIDPAC is bundled with Ref. [13], and is therefore publicly available. The SIDPAC software toolbox was developed at NASA Langley, and is continually expanded and improved. SIDPAC has been applied successfully to a wide variety of flight and wind tunnel experiments at NASA Langley and elsewhere, and is used at more than 80 institutions worldwide ${ }^{14}$.

\section{Aircraft Simulation}

This study was conducted in the NASA Langley Cockpit Motion Facility using the Integration Flight Deck simulator cockpit. Detailed discussions of the facility can be found in Refs. [15]-[16]. The cockpit is shown in Fig. 1. The simulation used for this work was a research implementation of a level D crew training flight simulation. The airplane simulated was a transcontinental-range commercial jet transport with twin engines mounted under the wing and a conventional, low, horizontal tail. Mass/inertia properties were representative of a mid-weight condition. For this study, the simulator was operated in a fixed-base mode, which did not use the hexapod motion base system.

\section{A. Flight Dynamics Model and Aerodynamic Database}

The six-degree-of-freedom simulation is a rigid-body model with quasi-static aeroelastic effects and Reynolds number scaling effects. Structural modes are not treated as independent states; rather, aeroelastic effects are accounted for by a series of dynamic pressure-based corrections that are applied to the rigid-body aerodynamic model. Reynolds number effects are incorporated directly into the aerodynamic database. The original crew training flight dynamics model was enhanced based on wind tunnel measurements to accurately represent the flight dynamic characteristic of a transport airplane at stall and post-stall flight conditions. The characteristics in the aerodynamic database are highly nonlinear with respect to relative wind incidence angles and bodyaxis rates at angles of attack near and above stall. This enhanced aerodynamic database is described in detail in Refs. [3]-[6].

\section{B. Flight Controls}

The flight control system used in this simulation study is a high-fidelity representation of a conventional hydromechanical flight control system for a transport airplane. A control column, wheel, and rudder pedals were used for primary flight control. These control inceptors were connected to a hydraulic control loader which accurately simulated the characteristics of a force-feel system for a transport airplane. The primary control surfaces are elevator,

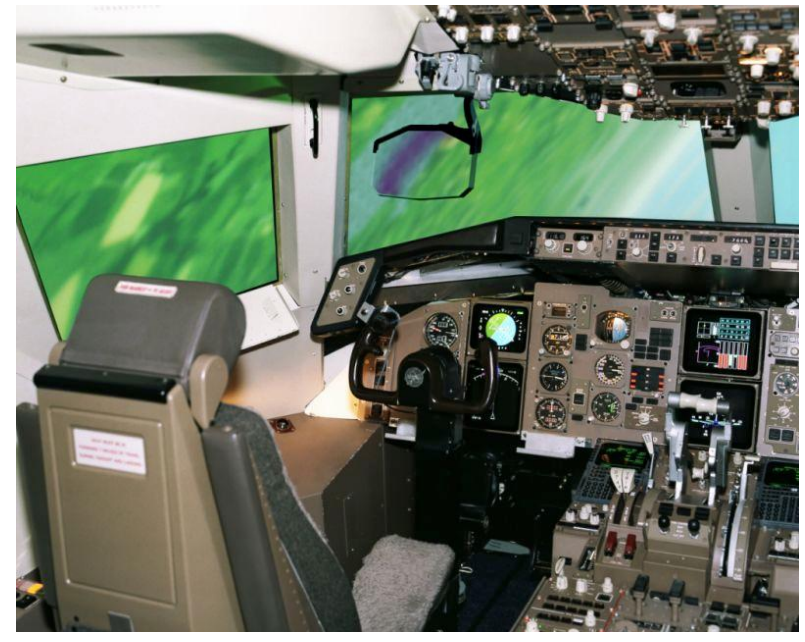

Figure 1. NASA Langley Integration Flight Deck (IFD) simulator cockpit

Credit: NASA Langley Research Center stabilizer, left and right ailerons, inboard and outboard left and right spoilers, and rudder. The aerodynamic effects of these control surfaces were modeled as increments to the baseline aerodynamic model. For the maneuvers in this study, the stabilizer was always kept at the constant initial deflection required for trim. Maneuvers were generally conducted with the yaw damper engaged, unless otherwise noted.

\section{Simulated Flight Data}

Aircraft sensor data was simulated by applying a sensor measurement noise model to the simulation outputs. Systematic instrumentation errors such as scale factor errors and biases were not applied, because typically these errors would be removed from measured flight data prior to modeling, using data compatibility analysis ${ }^{13}$. 
Similarly, simulated aircraft translational accelerations and air flow angles at the aircraft center of gravity were used, because flight data would be corrected in this way prior to modeling. Sensor noise levels were specified using estimated noise levels obtained by smoothing techniques applied to flight test data from similar maneuvers flown on a subscale transport aircraft ${ }^{10,11,13}$. Sensor noise levels are given in Table 1. Sampling rate for the simulated flight data was $50 \mathrm{~Hz}$.

\section{Piloted Flight Test Maneuvers}

For local aerodynamic modeling, a pilot would fly the aircraft to a reference flight condition, then execute a local perturbation maneuver by applying small perturbation inputs, starting from a steady reference flight condition. For global aerodynamic modeling, one conventional approach is to combine local aerodynamic modeling results obtained from many of these local perturbation maneuvers at different flight conditions. This requires numerous and accurate acquisitions of particular flight conditions, followed by the application of perturbation inputs at each condition, and finally combining the local results to produce a global model.

More efficient global aerodynamic modeling can be achieved by continuously applying multi-axis perturbation inputs while the aircraft flight condition is varied slowly. By changing the nominal angle of attack slowly, the maneuver can be considered a combination of informative multi-axis excitations for many different nominal angles of attack, executed in a single, efficient maneuver. The result is very informative flight data collected efficiently over a wide range of nominal angle of attack, which can be used for global aerodynamic modeling. This approach was implemented with automated multi-axis excitations while the pilot slowly changed flight condition in subscale aircraft flight tests $^{9-11}$. In the present work, the flight test maneuver was implemented completely by the pilot, using an approach analogous to what has been used successfully in the recent past for jet trainer flight tests ${ }^{12}$.

This section begins with a detailed description of how to fly an efficient flight test maneuver for global aerodynamic modeling. Following this, the data from a simulated flight test maneuver flown in this way is examined and evaluated for global aerodynamic modeling.

\section{A. Piloting Technique}

This section describes the piloting technique necessary to execute the flight test maneuvers that generated the simulated flight data for global aerodynamic modeling. The general idea is to excite the aircraft dynamics in all axes over a short time period by moving multiple control surfaces simultaneously in an uncorrelated manner. The excitation inputs should also have wideband frequency content over a range of frequencies encompassing the expected modal frequencies for the aircraft dynamic response. These excitation inputs are superimposed on a much slower input that slowly changes the flight condition from low angle of attack through stall/upset and recovery. The resulting simulated flight data exhibits low correlation among the explanatory variables, over a wide range of flight conditions, using a single, efficient piloted maneuver.

To fly the maneuver, the pilot first established idle thrust trimmed flight at a speed 20 percent over the stall speed, which put the airplane in a descent. The stabilizer trim system was configured to maintain a constant stabilizer position throughout the maneuver. The pilot then began applying small, uncorrelated perturbation inputs to the control column, control wheel, and rudder pedals. At the same time, the pilot slowly applied aft bias to the control column as necessary to establish a steady mean pitch attitude of 12.5 degrees, in order to obtain the desired airspeed deceleration rate of $2 \mathrm{ft} / \mathrm{s}$ per second. Mean pitch attitude was used to maintain a relatively steady airspeed deceleration (or steady mean angle of attack increase) because the pitch attitude varied slightly throughout the maneuver, as a result of the perturbation inputs. The pilot intentionally varied the frequency of the perturbations and made a conscious effort to avoid making correlated inputs, especially between control wheel and rudder pedal. The pilot varied the amplitude of the perturbations because stability and control characteristics changed during the maneuver as mean angle of attack progressed from low to higher values. The amplitudes of the perturbations were sized to obtain perturbation responses in pitch attitude and roll attitude of about \pm 2 degrees and \pm 5 degrees, respectively. Occasionally, the pilot applied momentary biases to the control wheel perturbations as necessary to maintain the desired trajectory and compensate for effects such as stall roll off.

A good process for implementing the maneuver is to first fly a standard slow approach to stall and recovery, in order to establish the feel and airspeed deceleration rate for the slowly-varying flight condition. Then a maneuver with similar mean trajectory can be flown, but this time with superimposed uncorrelated perturbation inputs. The requirements for a good maneuver are not stringent, so that a wide range of different maneuvers with many variations would be considered adequate for the global aerodynamic modeling described later. 


\section{B. Evaluating the Data from a Piloted Flight Test Maneuver}

Figure 2 shows simulated flight data from a piloted maneuver used to collect data for global aerodynamic modeling. Note the simultaneous excitation in all axes, and the slow change in flight condition from low angle of attack through high angle of attack, stall, and recovery.
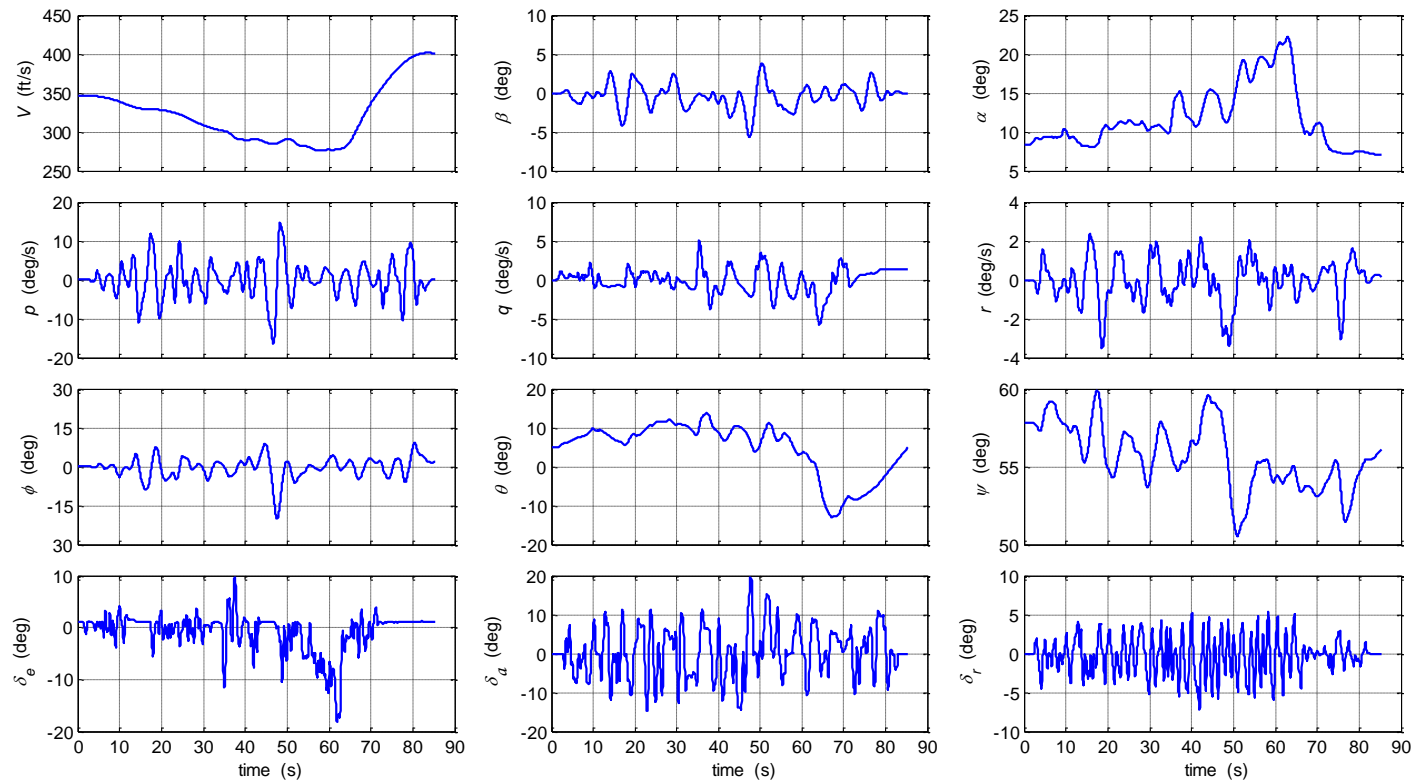

Figure 2. Simulated flight data for a global aerodynamic modeling maneuver

Figure 3 shows cross-plots of aircraft states and controls using data from the maneuver shown in Fig. 2. These plots demonstrate that a wide range of the explanatory variables generally used for aerodynamic modeling was swept through during this single maneuver. High (undesirable) pair-wise correlations would be indicated by lines or narrow ellipses in these plots, whereas nondescript shapes or wide ellipses, as seen in Fig. 3, indicate low (desirable) pair-wise correlations. Low pair-wise correlations mean that the aerodynamic dependencies on the explanatory variables can be identified accurately and without ambiguity. Cross-plots for other aircraft states and controls were similar in that the plots indicated low pair-wise correlations for the explanatory variables.

This novel maneuver exhibits a combination of low pair-wise correlations for the explanatory variables, multiaxis excitation, and slowly-varying flight condition covering large ranges of the explanatory variables to be used for aerodynamic modeling. These characteristics make the maneuver very effective and efficient for global aerodynamic modeling.

A series of these piloted maneuvers were flown on the fixed-base commercial jet transport aircraft simulator described in Section II. Each maneuver had a constant idle power setting and fixed aircraft configuration (flaps up, landing gear up), and all used multi-axis perturbation inputs implemented by the pilot, similar to what is shown in Figs. 2 and 3. 

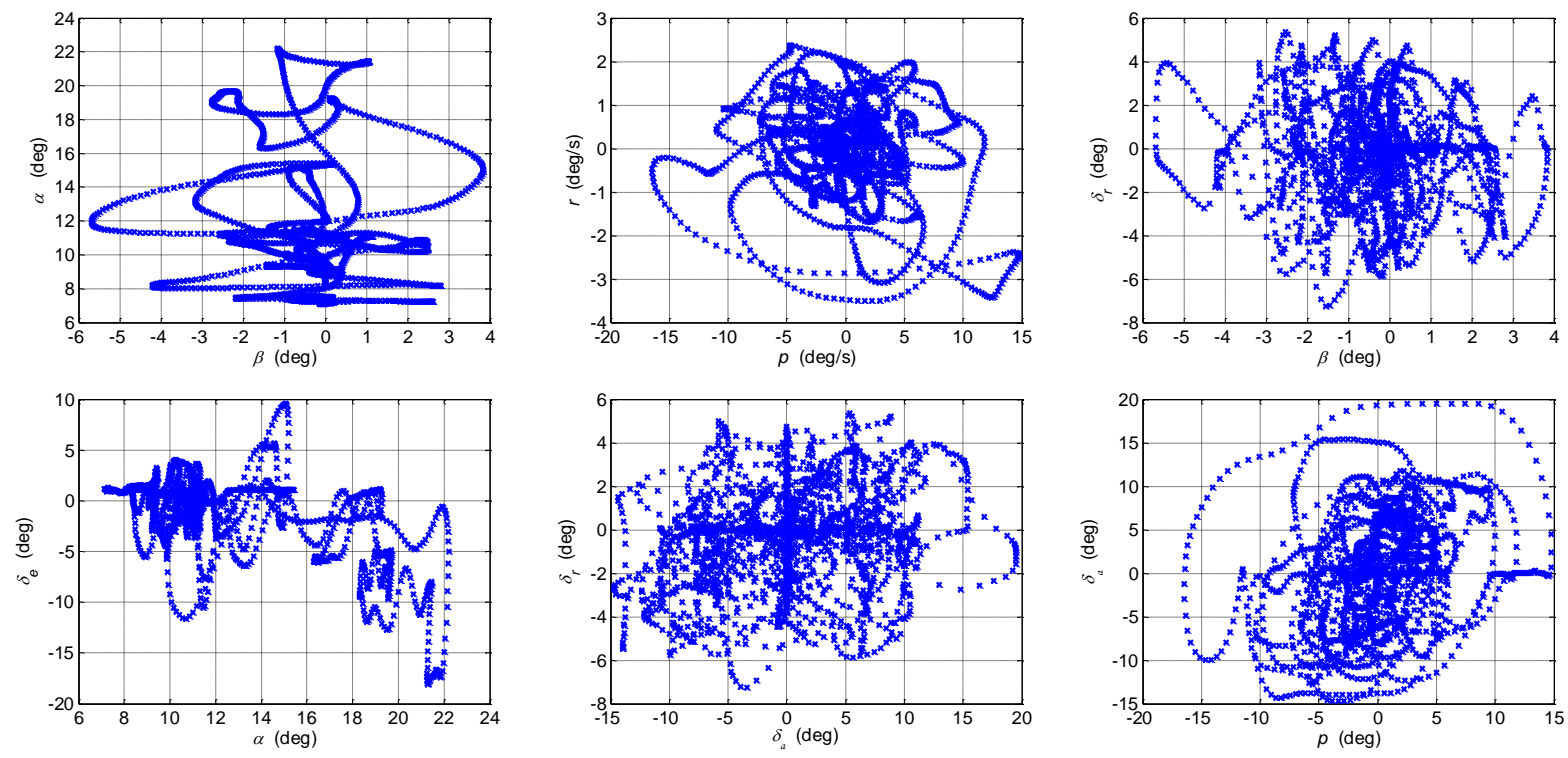

Figure 3. Simulated flight data cross plots for a global aerodynamic modeling maneuver

\section{Global Aerodynamic Modeling}

The objective of global aerodynamic modeling is to identify a model for each nondimensional aerodynamic force and moment coefficient as a function of explanatory variables that can be measured, such as angle of attack, pitch rate, and control surface deflections, over a large range of the explanatory variables. This requires two main tasks: 1) experiment design to collect dynamic data over a large range of the explanatory variables, which was addressed in Section III, and 2) identifying an accurate global model, which is the subject of this section.

In the typical approach to global aerodynamic modeling, a series of linear or simplified local models, which are valid for relatively small ranges of the explanatory variables, are joined together in some way to implement a global aerodynamic model. In another approach, a multivariate orthogonal function modeling technique can be used to identify global models, which are generally nonlinear and valid over relatively large ranges of the explanatory variables $^{17}$. However, for very large ranges of the explanatory variables, or severe local nonlinearities, this approach in some cases results in compromised local model fit in order to achieve a better global model fit. This is the result of using global orthogonal polynomial functions for the modeling, which do not have the ability to change locally without modifying the entire model. Other approaches have used data partitioning with simplified local model structures ${ }^{18}$ and localized modeling functions ${ }^{19}$ to address this problem, and also to make local model updates easier. However, these approaches require a procedure or analyst judgment for: 1) partitioning the explanatory variable space; 2) selecting the location of the local modeling functions in the explanatory variable space; and/or 3) selecting the mathematical structure of the local models.

In this work, the multivariate orthogonal function modeling developed in Ref. [17] was applied using both the explanatory variables and spline functions of the explanatory variables, as described in Ref. [11]. This approach provides the required local nonlinear modeling capability, while retaining easy physical interpretation of the model, and automating the selection of optimal modeling complexity necessary to accurately characterize the functional dependencies.

The next sections describe the approach to global aerodynamic modeling using multivariate orthogonal functions derived from multivariate polynomials and spline functions computed using measured explanatory variable data. Further details on this approach to global aerodynamic modeling can be found in Ref. [11].

\section{A. Aircraft Aerodynamic Modeling}

For global aerodynamic modeling from flight data, nondimensional aerodynamic force and moment coefficients are used as the response variable (also called the dependent variable) in the modeling problem. A separate modeling problem is solved for each force or moment coefficient, corresponding to minimizing the equation error in each 
individual equation of motion for the six rigid-body degrees of freedom of the aircraft. Values for the nondimensional aerodynamic force and moment coefficients cannot be measured directly in flight, but instead must be computed from measured and known quantities using the following equations ${ }^{13}$

$$
\begin{gathered}
C_{X} \equiv-C_{A}=\frac{\left(m a_{x}-X_{T}\right)}{\bar{q} S} \quad C_{Y}=\frac{m a_{y}}{\bar{q} S} \quad C_{Z}=-C_{N}=\frac{\left(m a_{z}-Z_{T}\right)}{\bar{q} S} \\
C_{D}=-C_{X} \cos \alpha-C_{Z} \sin \alpha \\
C_{l}=\frac{I_{x}}{\bar{q} S b}\left[\dot{p}-\frac{I_{x z}}{I_{x}}(p q+\dot{r})+\frac{\left(I_{z}-I_{y}\right)}{I_{x}} q r\right] \\
C_{m}=\frac{I_{y}}{\bar{q} S \bar{c}}\left[\dot{q}+\frac{\left(I_{x}-I_{z}\right)}{I_{y}} p r+\frac{I_{x z}}{I_{y}}\left(p^{2}-r^{2}\right)-M_{T}\right] \\
C_{n}=\frac{I_{z}}{\bar{q} S b}\left[\dot{r}-\frac{I_{x z}}{I_{z}}(\dot{p}-q r)+\frac{\left(I_{y}-I_{x}\right)}{I_{z}} p q\right]
\end{gathered}
$$

These equations retain the full nonlinear dynamics in the aircraft equations of motion.

The result is $N$ values of the nondimensional force and moment coefficients, where $N$ is the number of data points. These values are often called measured force and moment coefficients, even though they not measured directly, but rather computed from other measurements and known quantities. Mass/inertia properties are assumed to be known as a function of fuel state. Engine thrust and geometric properties such as wing span and wing reference area are assumed to be known. Dynamic pressure, translational accelerations, and explanatory variables such as angle of attack, sideslip angle, pitch rate, and control surface deflections, are measured directly. Angular accelerations are obtained by smoothed numerical differentiation of the measured angular rates ${ }^{13}$.

The desired form of the global aerodynamic model is a mathematical model structure with estimated model parameter values and associated uncertainties, relating the nondimensional aerodynamic force and moment coefficients to aircraft states and controls that can be measured.

\section{B. Multivariate Orthogonal Function Modeling}

The form of a multivariate orthogonal function model is

$$
z=a_{1} p_{1}+a_{2} p_{2}+\ldots+a_{n} p_{n}+\varepsilon
$$

where $z$ is an $N$-dimensional vector of the response variable (e.g., nondimensional force or moment coefficient), $z=\left[z_{1}, z_{2}, \ldots, z_{N}\right]^{T}$, modeled in terms of a linear combination of $n$ mutually orthogonal modeling functions $\boldsymbol{p}_{j}, j=1,2, \ldots, n$. Each $\boldsymbol{p}_{j}$ is an $N$-dimensional vector which in general depends on the explanatory variables. The $a_{j}, j=1,2, \ldots, n$ are constant model parameters to be determined, and $\boldsymbol{\varepsilon}$ denotes the modeling error vector.

Equation (6) represents a mathematical model used to represent functional dependencies in the measured data. The important questions of determining how the modeling functions $\boldsymbol{p}_{j}$ are computed from the explanatory variables, as well as which modeling functions should be included in Eq. (6), which implicitly determines $n$, will be addressed later. At this point, the properties of a multivariate orthogonal function model are examined.

Define an $N \times n$ matrix $\boldsymbol{P}$,

$$
\boldsymbol{P}=\left[\boldsymbol{p}_{1}, \boldsymbol{p}_{2}, \ldots, \boldsymbol{p}_{n}\right]
$$


and let $\boldsymbol{a}=\left[a_{1}, a_{2}, \ldots, a_{n}\right]^{T}$. Equation (6) can then be written as a standard least squares regression problem,

$$
z=\boldsymbol{P a}+\boldsymbol{\varepsilon}
$$

The error vector $\boldsymbol{\varepsilon}$ is to be minimized in a least squares sense. The goal is to determine $\boldsymbol{a}$ that minimizes the least squares cost function

$$
J=\frac{1}{2} \boldsymbol{\varepsilon}^{T} \boldsymbol{\varepsilon}=\frac{1}{2}(z-\boldsymbol{P a})^{T}(z-\boldsymbol{P a})
$$

The parameter vector estimate $\hat{\boldsymbol{a}}$ that minimizes this cost function is ${ }^{13}$

$$
\hat{\boldsymbol{a}}=\left[\boldsymbol{P}^{T} \boldsymbol{P}\right]^{-1} \boldsymbol{P}^{T} \boldsymbol{z}
$$

The estimated parameter covariance matrix is ${ }^{13}$

$$
\Sigma_{\hat{a}}=E\left[(\hat{\boldsymbol{a}}-\boldsymbol{a})(\hat{\boldsymbol{a}}-\boldsymbol{a})^{T}\right]=\sigma^{2}\left(\boldsymbol{P}^{T} \boldsymbol{P}\right)^{-1}
$$

where $E$ is the expectation operator, and the fit error variance $\sigma^{2}$ can be estimated from the residuals

$$
v=z-P \hat{a}
$$

using

$$
\hat{\sigma}^{2}=\frac{1}{(N-n)}\left[(z-\boldsymbol{P} \hat{\boldsymbol{a}})^{T}(z-\boldsymbol{P} \hat{\boldsymbol{a}})\right]=\frac{\boldsymbol{v}^{T} \boldsymbol{v}}{(N-n)}
$$

Parameter standard errors are computed as the square root of the diagonal elements of the $\Sigma_{\hat{\boldsymbol{a}}}$ matrix from Eq. (11), using $\hat{\sigma}^{2}$ from Eq. (13). The identified model output $\boldsymbol{y}$ is

$$
\boldsymbol{y}=\boldsymbol{P} \hat{\boldsymbol{a}}
$$

In conventional least-squares modeling, the modeling functions (columns of $\boldsymbol{P}$ ) are often polynomials in the explanatory variables. This approach corresponds to using the terms of a multivariate Taylor series expansion to approximate the functional dependence of the response variable on the explanatory variables. If the modeling functions are instead multivariate orthogonal functions generated from the explanatory variable data, it is easier to determine an appropriate model structure, because the explanatory capability of each modeling function is completely distinct from all the others. This decouples the least squares modeling problem, as will be shown now.

For mutually orthogonal modeling functions,

$$
\boldsymbol{p}_{i}^{T} \boldsymbol{p}_{j}=0 \quad, \quad i \neq j \quad, \quad i, j=1,2, \ldots, n
$$

and $\boldsymbol{P}^{T} \boldsymbol{P}$ is a diagonal matrix with the inner product of the orthogonal functions on the main diagonal. Using Eqs. (7) and (15) in Eq. (10), the $j^{\text {th }}$ element of the estimated parameter vector $\hat{\boldsymbol{a}}$ is computed as

$$
\hat{a}_{j}=\left(\boldsymbol{p}_{j}^{T} z\right) /\left(\boldsymbol{p}_{j}^{T} \boldsymbol{p}_{j}\right)
$$


Using Eqs. (7), (15), and (16) in Eq. (9),

$$
\hat{J}=\frac{1}{2}\left[z^{T} z-\sum_{j=1}^{n}\left(\boldsymbol{p}_{j}^{T} z\right)^{2} /\left(\boldsymbol{p}_{j}^{T} \boldsymbol{p}_{j}\right)\right]
$$

Equation (17) shows that when the modeling functions are orthogonal, the reduction in the least-squares cost function resulting from including the term $a_{j} \boldsymbol{p}_{j}$ in the model depends only on the response variable data $z$ and the added orthogonal modeling function $\boldsymbol{p}_{j}$. The least-squares modeling problem is therefore decoupled, which means each orthogonal modeling function can be evaluated independently in terms of its ability to reduce the least-squares model fit to the data, regardless of which other orthogonal modeling functions are already selected for the model. When the modeling functions $\boldsymbol{p}_{j}$ are instead polynomials in the explanatory variables (or any other non-orthogonal function set), the least-squares problem is coupled, and iterative analysis is required to select the modeling functions for an adequate model structure.

The orthogonal modeling functions to be included in the model are chosen to minimize predicted squared error, $P S E$, defined by ${ }^{20}$

$$
P S E \equiv \frac{(z-\boldsymbol{P} \hat{\boldsymbol{a}})^{T}(z-\boldsymbol{P} \hat{\boldsymbol{a}})}{N}+\sigma_{\max }^{2} \frac{n}{N}
$$

or

$$
P S E=\frac{2 \hat{J}}{N}+\sigma_{\max }^{2} \frac{n}{N}
$$

The constant $\sigma_{\max }^{2}$ is the upper-bound estimate of the squared error between future data and the model, i.e., the upper-bound mean squared error for prediction cases. The upper bound is used in the model over-fit penalty term to account for the fact that $P S E$ is calculated during the model structure determination stage, when the model structure is not correct. Using $\sigma_{\max }^{2}$ in the model complexity term also results in the PSE metric conservatively estimating the squared error for prediction cases.

A simple estimate of $\sigma_{\max }^{2}$ that is independent of the model structure can be obtained by considering $\sigma_{\max }^{2}$ to be the residual variance estimate for a constant model equal to the mean of the measured response values,

$$
\sigma_{\max }^{2}=\frac{1}{N-1} \sum_{i=1}^{N}\left[z_{i}-\bar{z}\right]^{2}
$$

where

$$
\bar{z}=\frac{1}{N} \sum_{i=1}^{N} z_{i}
$$


The PSE in Eq. (19) depends on the mean squared error, $2 \hat{J} / N$, and a term proportional to the number of terms in the model, $n$. The latter term prevents over-fitting the data with too many model terms, which is detrimental to model prediction accuracy ${ }^{13,20,21}$. While the mean squared error $2 \hat{J} / N$ must decrease with the addition of each orthogonal modeling function to the model (by Eq. (17)), the over-fit penalty term $\sigma_{\max }^{2} n / N$ must increase with each added model term (increased $n$ ). Introducing the orthogonal modeling functions into the model in order of most effective to least effective in reducing the mean squared error (quantified by $(2 / N)\left(\boldsymbol{p}_{j}^{T} z\right)^{2} /\left(\boldsymbol{p}_{j}^{T} \boldsymbol{p}_{j}\right)$ for the $j^{\text {th }}$ orthogonal modeling function) results in the PSE metric always having a single global minimum.

Figure 4 depicts this graphically, using actual modeling results from Ref. [21]. The figure shows that after the first 6 modeling functions, the added model complexity associated with an additional orthogonal modeling function is not justified by the associated reduction in mean squared error. This point is marked by minimum PSE, which defines an adequate model

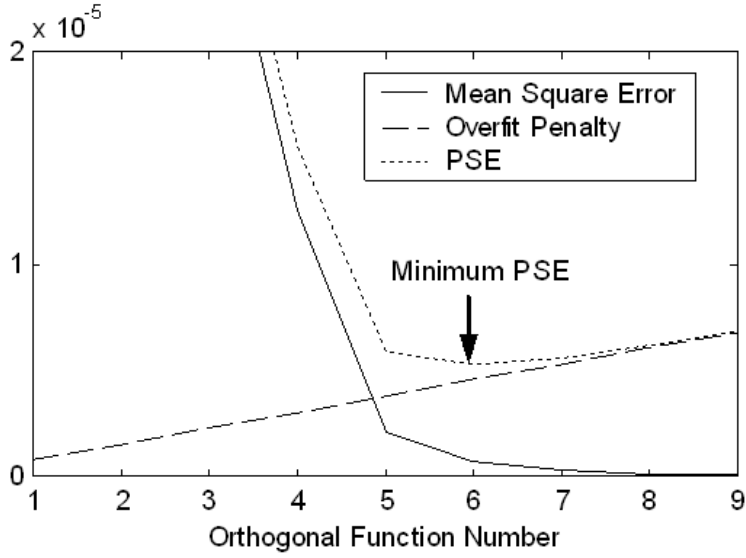

Figure 4. Model structure determination using orthogonal functions and PSE structure with good predictive capability. Ref. [20] contains further statistical arguments and analysis for the form of PSE given in Eq. (18), including justification for its use in modeling problems.

Using orthogonal functions to model the response variable makes it possible to evaluate the merit of including each modeling function individually, using the predicted squared error PSE. The goal is to select a model structure with minimum PSE, and the PSE always has a single global minimum for orthogonal modeling functions. This makes the model structure determination a well-defined and straightforward process that can be (and was) automated.

\section{Generating Orthogonal Modeling Functions}

Multivariate orthogonal functions can be generated from ordinary multivariate functions in the explanatory variables using a Gram-Schmidt orthogonalization procedure. This approach is described in Refs. [11], [13], [17], and [21], which are the basis for the material presented here.

The process begins by choosing one of the ordinary multivariate functions as the first orthogonal function. Typically, a vector of ones (associated with the bias term in the model) is chosen as the first orthogonal function,

$$
p_{1}=1
$$

In general, any function of the explanatory variables can be chosen as the first orthogonal function, without any change in the procedure. To generate the next orthogonal function, an ordinary multivariate polynomial function is made orthogonal to the preceding orthogonal function(s). Define the $j^{\text {th }}$ orthogonal function $\boldsymbol{p}_{j}$ as

$$
\boldsymbol{p}_{j}=\boldsymbol{\xi}_{j}-\sum_{k=1}^{j-1} \gamma_{k j} \boldsymbol{p}_{k} \quad j=2,3, \ldots, n_{t}
$$

where $\xi_{j}$ is the $j^{\text {th }}$ ordinary multivariate function vector. For example, each $\xi_{j}$ could be some ordinary polynomial function of the explanatory variables, or spline functions of the explanatory variables, or multiplicative combinations of these, or any general multivariate function. The $\gamma_{k j}$ for $k=1,2, \ldots, j-1$ are scalars determined by multiplying both sides of Eq. (23) by $\boldsymbol{p}_{k}^{T}$, then invoking the mutual orthogonality of the $\boldsymbol{p}_{k}, k=1,2, \ldots, j$, and solving for $\gamma_{k j}$, 


$$
\gamma_{k j}=\frac{\boldsymbol{p}_{k}^{T} \boldsymbol{\xi}_{j}}{\boldsymbol{p}_{k}^{T} \boldsymbol{p}_{k}} \quad k=1,2, \ldots, j-1
$$

The same process can be implemented in sequence for each ordinary multivariate function $\xi_{j}, j=2,3, \ldots, n_{t}$. The total number of ordinary multivariate functions used as raw material for generating the multivariate orthogonal functions, including the bias term, is $n_{t}$. It can be seen from Eqs. (22)-(24) that each orthogonal function can be expressed exactly in terms of a linear expansion of the original multivariate functions. The orthogonal functions are generated sequentially by orthogonalizing the original multivariate functions with respect to the orthogonal functions already computed. Each orthogonal function can be considered an orthogonalized version of an original multivariate function.

The multivariate orthogonal function generation method described here normally starts by generating all possible ordinary multivariate polynomials in the explanatory variables, up to a selected maximum order. For example, when modeling the nondimensional vertical force coefficient $C_{Z}$, the explanatory variables might be angle of attack $\alpha$, nondimensional pitch rate $\hat{q} \equiv q \bar{c} / 2 V$, and elevator deflection $\delta_{e}$. If the selected maximum order is 3 , then the ordinary multivariate polynomial modeling functions used as the raw material for the orthogonalization process would include terms like $\alpha, \hat{q}, \delta_{e}, \alpha^{2}, \delta_{e}^{3}, \alpha \hat{q}^{2}, a \hat{q} \delta_{e}$, etc. Note that considering any other candidate explanatory variables, such as sideslip angle $\beta$, can be done by simply including the sideslip angle among the group of explanatory variables. If it turns out that the sideslip angle is not needed to model $C_{Z}$, the model structure determination using orthogonal modeling functions will not select any orthogonal functions associated with sideslip angle. This occurs automatically in the course of the model structure determination process described earlier. Therefore, there is no harm in including explanatory variables that might not be important, except that additional computation time will be required to identify the model structure, because additional multivariate orthogonal functions will be generated and sorted. Similarly, if the maximum order is chosen higher than necessary, the only penalty would be the increased computation time necessary for generating and sorting the additional orthogonal functions. The final identified model would be the same. Consequently, the choices that the analyst needs to make are easy and not critical to the quality of the final modeling results.

If the $\boldsymbol{p}_{j}$ vectors and the $\boldsymbol{\xi}_{j}$ vectors are arranged as columns of matrices $\boldsymbol{P}$ and $\boldsymbol{X}$, respectively, and the $\gamma_{k j}$ are elements in the $k^{\text {th }}$ row and $j^{\text {th }}$ column of an upper triangular matrix $\boldsymbol{G}$ with ones on the diagonal,

$$
\boldsymbol{G}=\left[\begin{array}{ccccc}
1 & \gamma_{12} & \gamma_{13} & \cdots & \gamma_{1 n_{t}} \\
0 & 1 & \gamma_{23} & \cdots & \gamma_{2 n_{t}} \\
0 & 0 & 1 & \cdots & \gamma_{3 n_{t}} \\
\vdots & \vdots & \vdots & \vdots & \vdots \\
0 & 0 & 0 & \cdots & 1
\end{array}\right]
$$

Then

$$
\boldsymbol{X}=\boldsymbol{P} \boldsymbol{G}
$$

which leads to

$$
\boldsymbol{P}=\boldsymbol{X} \boldsymbol{G}^{-1}
$$

The columns of $\boldsymbol{G}^{-1}$ contain the coefficients for expansion of each column of $\boldsymbol{P}$ (i.e., each multivariate orthogonal function) in terms of an exact linear expansion in the original multivariate functions in the columns of $\boldsymbol{X}$. Equation (27) can be used to express each multivariate orthogonal function in terms of the original multivariate functions. The manner in which the orthogonal functions are generated allows them to be decomposed without ambiguity into an expansion of the original multivariate functions, which have physical meaning. 


\section{Conversion to Physically Meaningful Multivariate Function Models}

After the model structure is determined using multivariate orthogonal modeling functions for minimum PSE, the identified model output is

$$
\boldsymbol{y}=\boldsymbol{P} \hat{\boldsymbol{a}}
$$

where the $\boldsymbol{P}$ matrix now includes only the $n$ orthogonal functions selected in the model structure determination, $n \leq n_{t}$. Each retained orthogonal modeling function can be decomposed without error into an expansion of the original multivariate functions in the explanatory variables, using the columns of $\boldsymbol{G}^{-1}$ in Eq. (27) corresponding to the retained orthogonal functions. Common terms are combined using double precision arithmetic to arrive finally at a model using only original multivariate functions in the explanatory variables. Terms that contribute less than 0.1 percent of the final model root-mean-square magnitude are dropped.

The final form of the model is a sum of ordinary multivariate functions in the explanatory variables, with associated model parameter estimates and uncertainties.

\section{E. Including Spline Functions in the Orthogonalization}

For global aerodynamic modeling, functional dependencies of the aerodynamic coefficients on the explanatory variables can exhibit significant localized variation or changes in character. In these cases, a global polynomial model can be inadequate for capturing the local variations. To solve this problem, spline functions in the explanatory variables can be introduced as additional explanatory variables. Splines have local modeling capability that global polynomial modeling functions do not have, because splines are polynomials defined only on selected intervals. Low-order polynomial terms defined on limited intervals can approximate local nonlinearities quite well. Using polynomial splines also retains clear physical interpretation in the final model.

Spline functions are defined as piecewise polynomials functions of degree $m$ in one or more explanatory variables. The term "piecewise" means that the polynomial is different for specific ranges of the explanatory variables. Spline function values and derivatives agree at the points where the piecewise polynomials join. These points are called knots, and are defined as specific values of each explanatory variable. A polynomial spline $S_{m}(x)$ of degree $m$ with continuous derivatives up to degree $m-1$, for a single explanatory variable $x \in\left[x_{\min }, x_{\max }\right]$, can be expressed as

$$
S_{m}(x)=\sum_{r=1}^{m} C_{r} x^{r}+\sum_{i=1}^{k} D_{i}\left(x-x_{i}\right)_{+}^{m}
$$

where

$$
\left(x-x_{i}\right)_{+}^{m}=\left\{\begin{array}{cc}
\left(x-x_{i}\right)^{m} & x>x_{i} \\
0 & x \leq x_{i}
\end{array}\right.
$$

and the $C_{r}$ and $D_{i}$ are constants and $\left(x-x_{i}\right)_{+}^{m}$ are the piecewise parts of the spline function. The values $x_{1}, x_{2}, \ldots, x_{k}$ are knots which satisfy the condition

$$
x_{\min }<x_{1}<x_{2}<\ldots<x_{k}<x_{\max }
$$

Three piecewise spline types are sketched in Fig. 5 for the same selected knots in angle of attack.

Note that when the spline knots are the same, a higher-order piecewise spline in a single explanatory variable can be computed as a multiplication of lower-order piecewise splines in the same explanatory variable,

$$
\left(x-x_{i}\right)_{+}^{m}=\left(x-x_{i}\right)_{+}^{m-1}\left(x-x_{i}\right)_{+}^{1} \quad m \geq 1
$$


This fact can be used advantageously in generating multivariate orthogonal modeling functions with excellent local modeling capability. An example will be used to demonstrate the idea.

Suppose that the nondimensional vertical aerodynamic force coefficient $C_{Z}$ is being modeled with explanatory variables angle of attack $\alpha$ and elevator control deflection $\delta_{e}$. For a maximum selected model order 2 , the ordinary polynomial modeling functions that will serve as raw material for the orthogonalization process are:

$$
1, \alpha, \alpha^{2}, \alpha \delta_{e}, \delta_{e}, \delta_{e}^{2}
$$

which would lead to a multivariate polynomial model of the form

$$
C_{Z}=C_{Z_{o}}+C_{Z_{\alpha}} \alpha+C_{Z_{\alpha^{2}}} \alpha^{2}+C_{Z_{\alpha \delta_{e}}} \alpha \delta_{e}+C_{Z_{\delta_{e}}} \delta_{e}+C_{Z_{\delta_{e}^{2}}} \delta_{e}^{2}
$$

where the values of the model parameters such as $C_{Z_{o}}$ and $C_{Z_{\alpha \delta_{e}}}$ would be estimated from the data using the orthogonal function modeling and subsequent decomposition procedures described earlier. Some of the terms on the right side of Eq. (34) might not be present, depending on the results of the model structure determination using orthogonal functions. This represents a global polynomial model.

Now introduce a first-order spline in angle of attack with a single knot located at $10 \mathrm{deg}$ as an additional explanatory variable. Using the same maximum model order 2 , the set of ordinary polynomial modeling functions expands to

$$
1, \alpha, \alpha^{2}, \alpha \delta_{e}, \delta_{e}, \delta_{e}^{2},(\alpha-10)_{+}^{1}, \alpha(\alpha-10)_{+}^{1}, \delta_{e}(\alpha-10)_{+}^{1},(\alpha-10)_{+}^{2}
$$

which would lead to a multivariate polynomial model of the form

$$
\begin{aligned}
C_{Z}= & C_{Z_{o}}+C_{Z_{\alpha}} \alpha+C_{Z_{\alpha^{2}}} \alpha^{2}+C_{Z_{\alpha \delta_{e}}} \alpha \delta_{e}+C_{Z_{\delta_{e}}} \delta_{e}+C_{Z_{\delta_{e}^{2}}} \delta_{e}^{2} \\
& +C_{Z_{\alpha_{10}^{1}}}(\alpha-10)_{+}^{1}+C_{Z_{\alpha \alpha_{10}^{1}}} \alpha(\alpha-10)_{+}^{1}+C_{Z_{\delta_{e} \alpha_{10}^{1}}} \delta_{e}(\alpha-10)_{+}^{1}+C_{Z_{\alpha_{10}^{2}}}(\alpha-10)_{+}^{2}
\end{aligned}
$$

As before, some of the terms on the right side of Eq. (36) might be not be present, depending on the results of the model structure determination using orthogonal functions.

With this expanded set of ordinary modeling functions as raw material, more orthogonal functions will be generated, and there is now capability to accommodate changes in the linear dependence of $C_{Z}$ on $\alpha$ for $\alpha>10 \mathrm{deg}$, as well as additional $\alpha^{2}$ and $\alpha \delta_{e}$ nonlinearities that only take effect when $\alpha>10 \mathrm{deg}$. Note also that $\alpha(\alpha-10)_{+}^{1}$ and $(\alpha-10)_{+}^{2}$, for example, are in general not the same function. Because higher-order splines are created automatically by the multiplication of first-order splines (cf. Eq. (32)), only first-order splines in the explanatory variables need to be included as additional explanatory variables.

Extrapolating from this simple example, it is clear that multiple knots in the explanatory variables would provide local nonlinear modeling capability in multiple explanatory variables, while retaining physical insight into the functional dependencies. The automated orthogonalization and sorting process described earlier identifies which nonlinear terms are necessary to
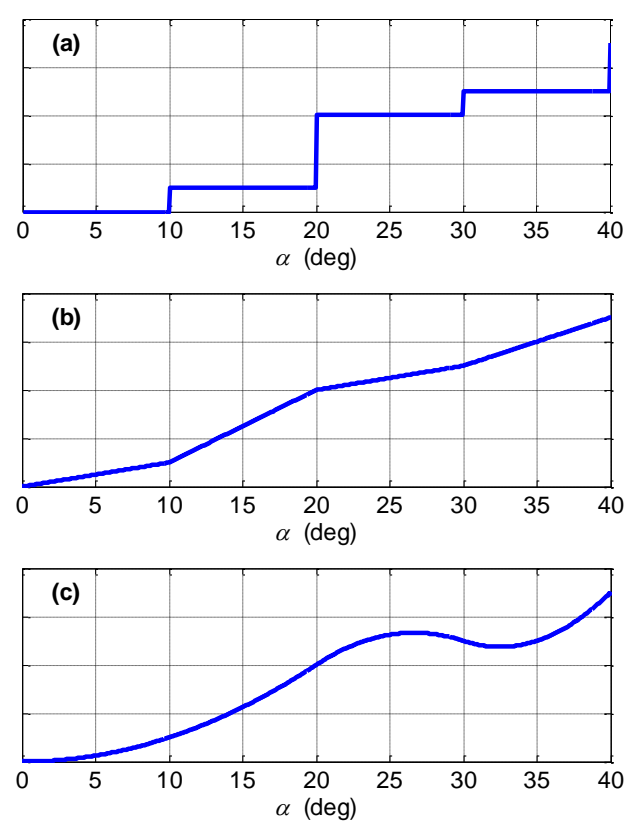

Figure 5. Polynomial splines in angle of attack: (a) zero-degree, (b) first-degree, (c) second-degree 
characterize the functional dependencies and estimates the associated model parameters and associated uncertainties. Inputs required from the analyst relate only to the limits of what should be considered, such as which explanatory variables to consider, maximum model order, and knot locations. However, these can be specified very generously, because the orthogonal function modeling algorithm automatically sorts out which of the terms are important, based on the data, and discards the rest. The result is a global parsimonious model with excellent global and local nonlinear modeling capability and easy physical interpretation.

When many different splines are considered for closely-spaced knot locations, perhaps for more than one explanatory variable, it is possible to run into memory limitations for the large number of orthogonal functions that are generated. This problem can be avoided by considering subsets of the full set of splines during a series of identification stages, then retaining only the selected spline terms at successive stages. This amounts to a sequential search for effective modeling terms. For example, the first stage might consider only first-order and second-order splines in angle of attack, with knots spaced 1 deg apart, from 8 deg to 22 deg. The model structure determination might then select only first-order spline terms at angle of attack knot locations of $16 \mathrm{deg}$ and $20 \mathrm{deg}$. The next stage would retain all of the terms identified for the model in the first stage, but might investigate first-order and secondorder splines in sideslip angle from -10 deg to $10 \mathrm{deg}$ with knots spaced $1 \mathrm{deg}$ apart, for example, and so on. The general idea is that once a candidate nonlinear or spline modeling function is discarded at any stage, it need not be considered again. This saves computation and memory during the model structure identification process, while still allowing a wide range of nonlinear and spline modeling terms to be considered for inclusion in the final model.

\section{Results}

The techniques for piloted flight test maneuvers and global aerodynamic modeling described in Sections III and IV were applied using the fixed-base commercial jet transport aircraft simulator described in Section II. Data from a typical piloted maneuver is shown in Figs. 2 and 3.

\section{A. Identified Models and Comparison with the Simulation Database}

Applying the flight testing and global modeling techniques in simulation allows comparison of the global aerodynamic models identified from the simulated flight test data with the known underlying aerodynamic database used in the simulation. The aerodynamic database in the simulation was implemented in tabular form, based on wind tunnel data. Simulated flight data from the piloted maneuver shown in Figures 2 and 3 was used initially for the global aerodynamic modeling. Results showed accurate characterization at low angles of attack, but inadequate characterization of the relatively complex functional dependencies near stall. It was found that the modeling near stall could be improved if an additional stall maneuver without pilot excitation was included in the data used for global modeling. The additional maneuver provided enhanced data information content for a more detailed and accurate global model near stall. Figure 6 shows the stall maneuver that was combined with the data from Figs. 2 and 3 to generate the modeling results shown in this section.

For the global aerodynamic modeling, initial modeling was done using coarse spacing of knot locations in each explanatory variable to identify which explanatory variables required the increased local nonlinear modeling capability provided by spline terms. This analysis showed that the global aerodynamic modeling required spline terms mainly for angle of attack. First-order splines in angle of attack with knots finely spaced at 1 deg intervals between $12 \mathrm{deg}$ and $22 \mathrm{deg}$ inclusive (knots = [12:1:22] deg) were added as additional explanatory variables for the global aerodynamic modeling. These terms were culled from a larger candidate pool of first-order splines in angle of attack with more knot locations, using the automated orthogonal function modeling process. For each nondimensional force and moment coefficient model, model terms with up to second-order complexity were orthogonalized and sorted to identify an adequate model structure and estimate the associated model parameter values and uncertainties. This upper-bound second-order complexity applied to the conventional explanatory variables (such as angle of attack, nondimensional pitch rate, and elevator control deflection), as well as the firstorder splines in angle of attack, which were included as additional explanatory variables. The second-order complexity automatically included up to second-order multiplicative combinations of these functions. Because the first-order spline terms were multiplied by themselves and each other, up to second order, this approach allowed for up to second-order spline terms in angle of attack. Other modeling terms considered in the model identification process included nonlinear cross terms invoked at specific values of the angle of attack knots, such as $(\alpha-10)_{+}^{1} \delta_{e}$

or $(\alpha-12)_{+}^{1}(q \bar{c} / 2 V)$. As expected, only some of all the candidate model terms were selected for inclusion in the global model for each aerodynamic coefficient. 

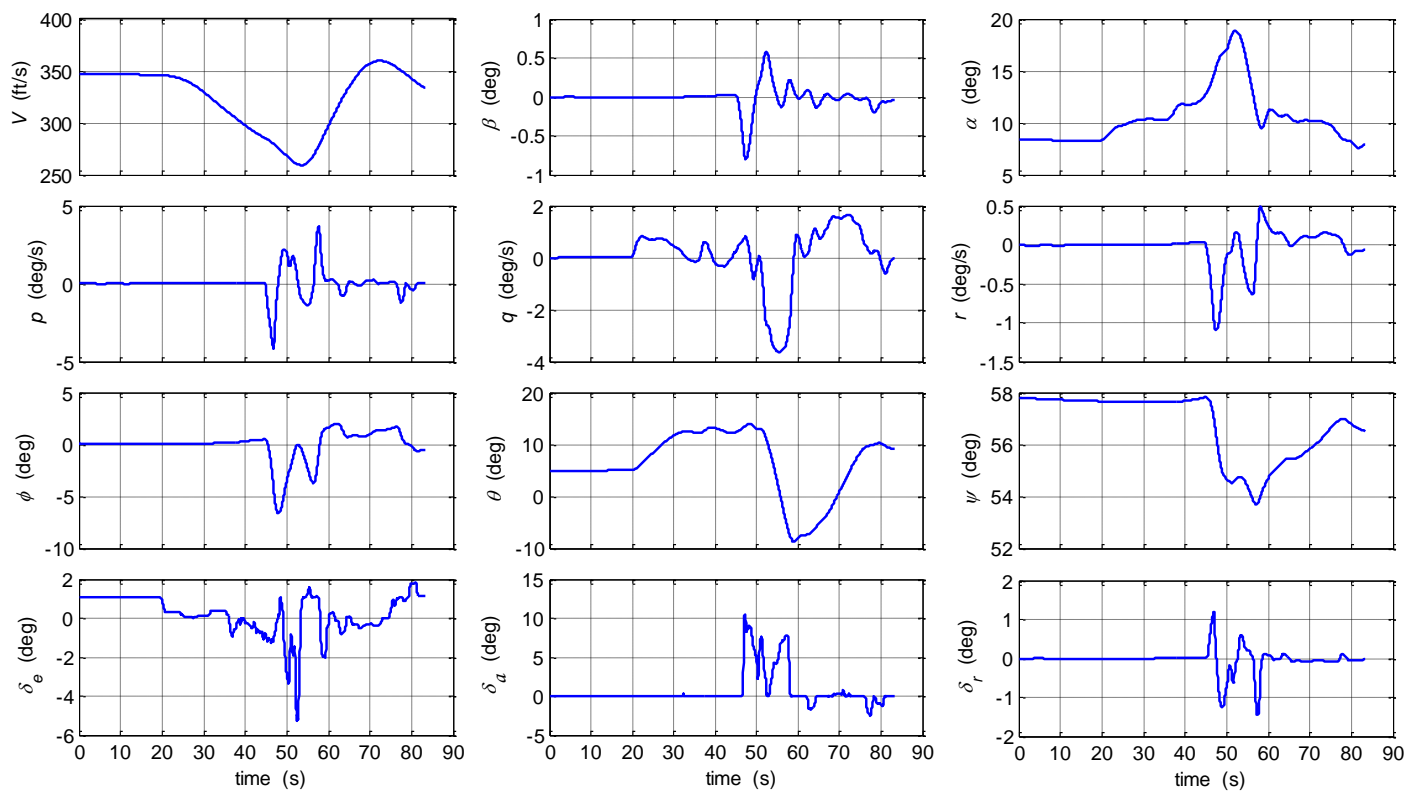

Figure 6. Simulated flight data for a stall maneuver

Subsequent to the angle of attack spline term identification, a first-order spline in sideslip angle with knot location at $0 \mathrm{deg}$, and zero-order splines in angle of attack with knot locations at 12.4, 12.6, and 12.8 deg were identified as important model terms for some of the aerodynamic coefficient models. These additional spline terms were identified in a sequential fashion, as described earlier, and were selected from a pool of candidates. It was found that additional candidate model terms could be supplied liberally and sequentially to the automated orthogonal function modeling procedure, with the result that only important and statistically significant model terms would be selected and retained in the model.

The overall approach permits simple specification of allowable model complexity that results in a remarkably diverse and powerful set of candidate modeling terms, from which an adequate model structure is selected automatically based on data information content, orthogonalization, and statistical modeling metrics, as explained in Section IV.

Figure 7 shows three-dimensional plots for all six aerodynamic coefficients, comparing the simulated flight data (x markers) with a mesh surface drawn by interrogating the aerodynamic database in the simulation, and a smooth surface drawn using the global aerodynamic model identified from simulated flight data alone. The ranges of the explanatory variables used to generate the mesh surface and the smooth surface were chosen to include all of the simulated flight data. Note that numbers on the vertical scales had to be removed because of proprietary restrictions on the wind-tunnel database. The results in Fig. 7 show that for all aerodynamic coefficients, the smooth surface and mesh surface are very similar, indicating that the global aerodynamic modeling approach captured the underlying functional dependencies accurately and efficiently, for all six aerodynamic coefficients simultaneously, using data from just two flight test maneuvers. The modeling technique successfully identified complex local nonlinearity, as shown in Fig. 7.

Close inspection shows only small mismatches between the smooth surface and the mesh surface at locations relatively far from the simulated flight data, which would be areas of extrapolation for the identified global model. Flying another maneuver through those parts of the explanatory variable subspace would provide the data necessary to correct the small mismatches. The three-dimensional plots shown in Fig. 7 must necessarily omit some of the explanatory variables (because there are more than two explanatory variables, and the third dimension is used for the response variable), so the mismatch between simulated flight data and the smooth and mesh surfaces represents mostly dependence on explanatory variables that cannot be shown on a three-dimensional plot. 

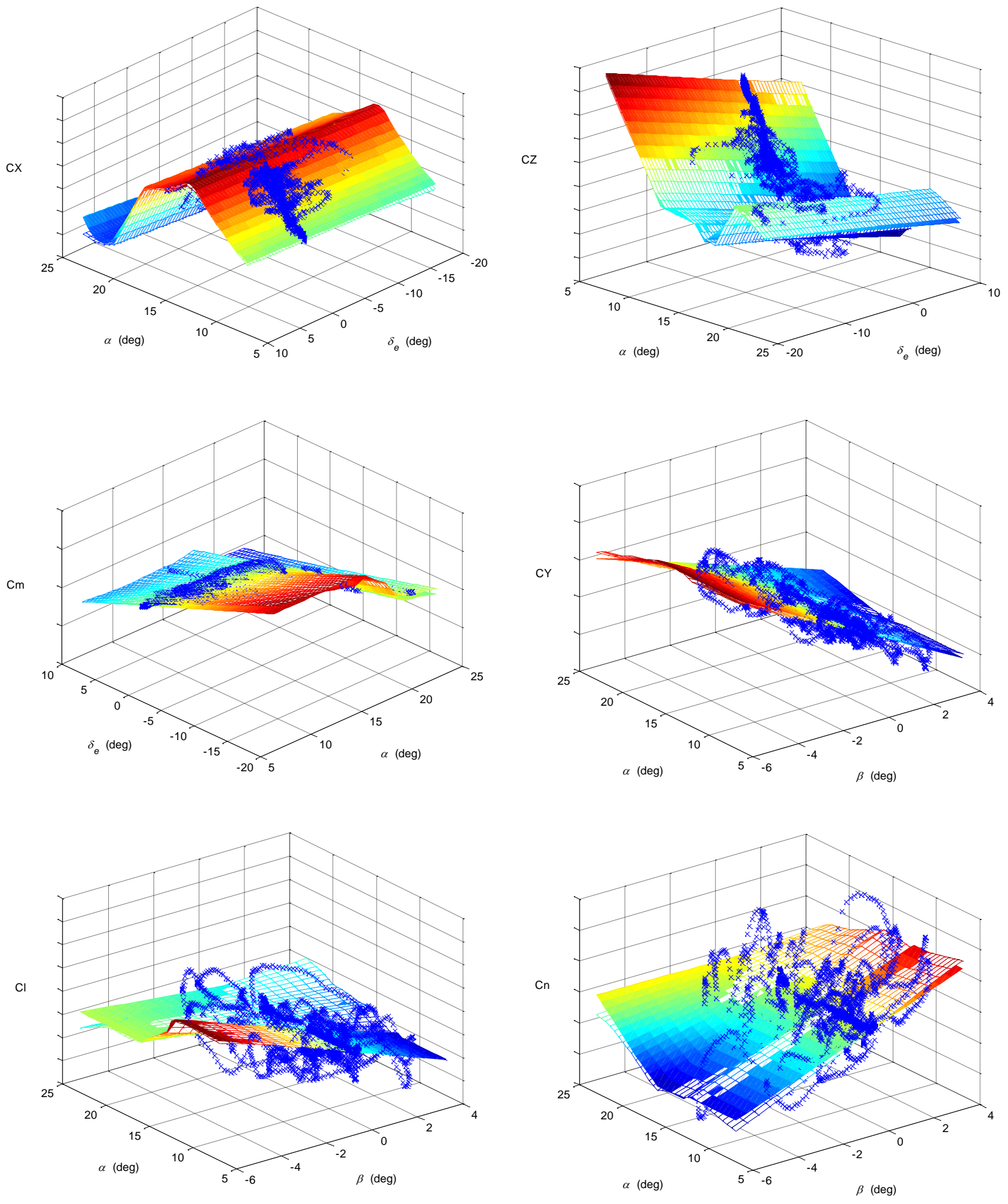

Figure 7. Global aerodynamic models identified from simulated flight data

The results shown in Fig. 7 suggest that a few well-flown maneuvers of the type described here could very effectively cover a large portion of the explanatory variable subspace, and when combined with the orthogonal function modeling procedure, could be used in place of wind-tunnel testing that requires extensive grids of test points to generate the aerodynamic database. Because the modeling uses an equation-error least-squares 
formulation, identifying a model based on combined data from multiple maneuvers is straightforward, and is done by simply stacking the data from multiple maneuvers into a single larger problem ${ }^{22}$.

As an example of the compact and analytical final form of the global model, the global model identified for nondimensional aerodynamic vertical force coefficient $C_{Z}$ from simulated flight data was

$$
\begin{aligned}
C_{Z}= & C_{Z_{o}}+C_{Z_{\alpha}} \alpha+C_{Z_{\alpha_{13}^{1}}}(\alpha-13)_{+}^{1}+C_{Z_{\alpha_{12.4}^{0}}}(\alpha-12.4)_{+}^{0}+C_{Z_{\alpha \delta_{e}}} \alpha \delta_{e}+C_{Z_{\alpha_{14}^{1}}}(\alpha-14)_{+}^{1} \\
& +C_{Z_{\alpha_{12.8}^{0}}}(\alpha-12.8)_{+}^{0}+C_{Z_{\alpha_{20}^{1}}}(\alpha-20)_{+}^{1}+C_{Z_{\alpha q}} \alpha \frac{q \bar{c}}{2 V}+C_{Z_{\alpha_{16}^{1}}}(\alpha-16)_{+}^{1}+C_{Z_{\delta_{e}}} \delta_{e} \\
& +C_{Z_{q \alpha_{12}^{1}}} \frac{q \bar{c}}{2 V}(\alpha-12)_{+}^{1}+C_{Z_{q}} \frac{q \bar{c}}{2 V}+C_{Z_{\alpha_{19}^{1}}}(\alpha-19)_{+}^{1}
\end{aligned}
$$

where model parameter estimates and uncertainties cannot be given, because of proprietary data restrictions. Although data restrictions significantly compromised the presentation of results in this work, the fact that the windtunnel database is considered highly realistic ${ }^{3-6}$ was judged to be more important. Showing that the piloted maneuvers and global modeling techniques can be successfully applied in simulation using this realistic database is a strong indicator of potential success for application to real flight testing of large commercial jet transport aircraft.

\section{B. Prediction Testing}

The prediction capability of the identified global aerodynamic models was tested using simulated flight data from maneuvers that were not used in the global model identification. Figure 8 shows data from a simulated slow approach to stall without pilot excitations, with the yaw damper on. The predictions shown in Fig. 8 are remarkably good, considering that data from only two flight test maneuvers was used to identify the global models. Figure 9 shows the prediction for a similar slow approach to stall maneuver, but with the yaw damper turned off. In this case, there is some degradation in the prediction, particularly for the yawing moment coefficient near stall. This is likely because the explanatory variables for this maneuver (where yaw damper was off) ranged beyond the
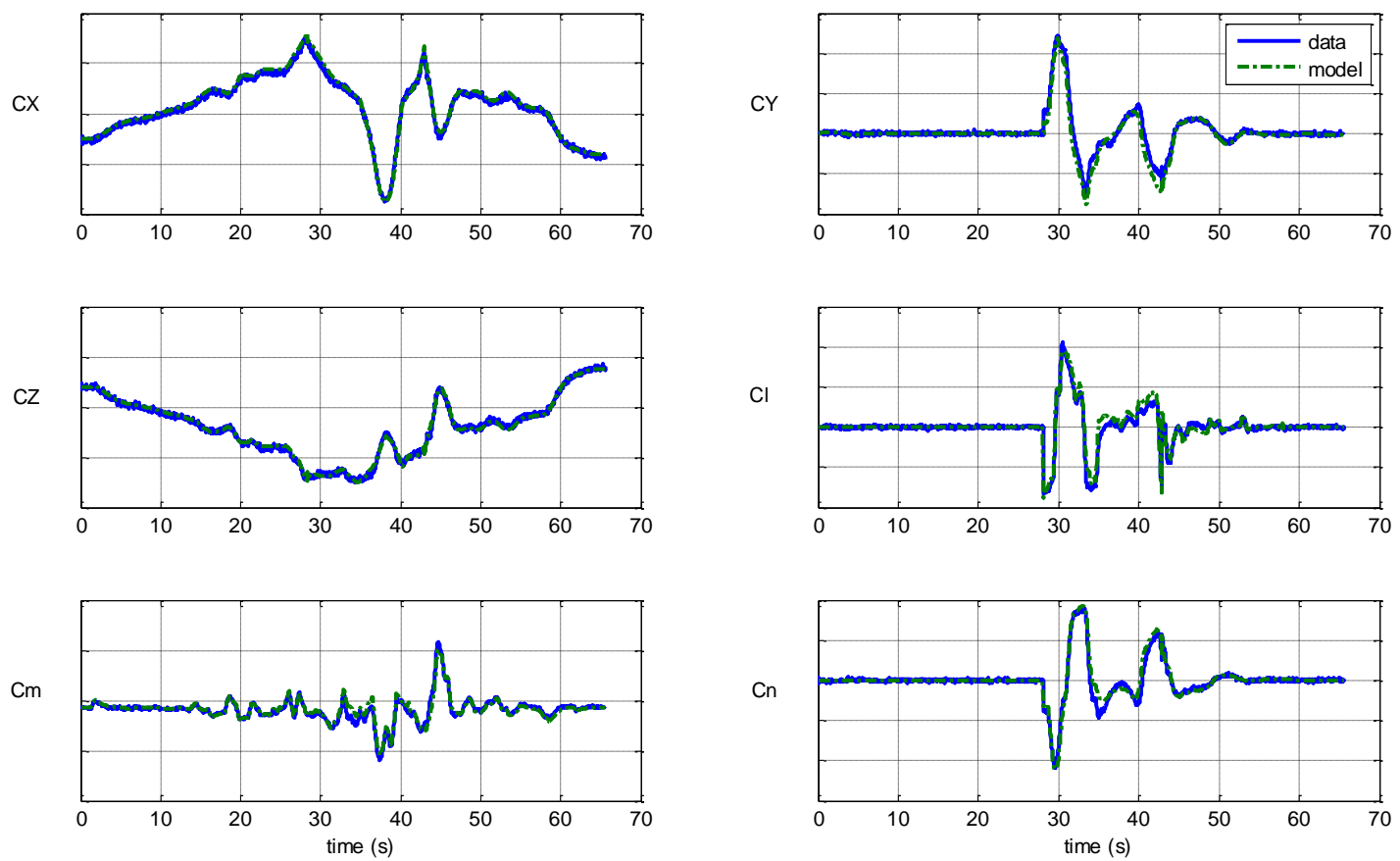

Figure 8. Stall maneuver prediction, yaw damper on 
subspace covered by the explanatory variables for the identification data (where yaw damper was on), making this prediction case at least a partial extrapolation for the lateral coefficients. Note that the quality of the longitudinal coefficient predictions in Fig. 9 was similar to that shown in Fig. 8, because the yaw damping affects mainly lateral explanatory variables. For both of the maneuvers shown in Figs. 8 and 9, the root-mean-square prediction error was less than 0.01 for the longitudinal coefficients $\left(C_{X}, C_{Z}, C_{m}\right)$, and less than 0.001 for the lateral coefficients $\left(C_{Y}, C_{1}, C_{n}\right)$. Note that these prediction errors included the measurement noise.

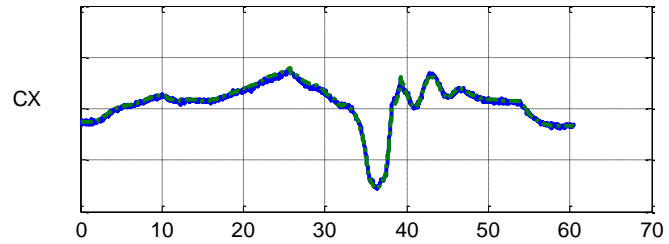

$\mathrm{CY}$
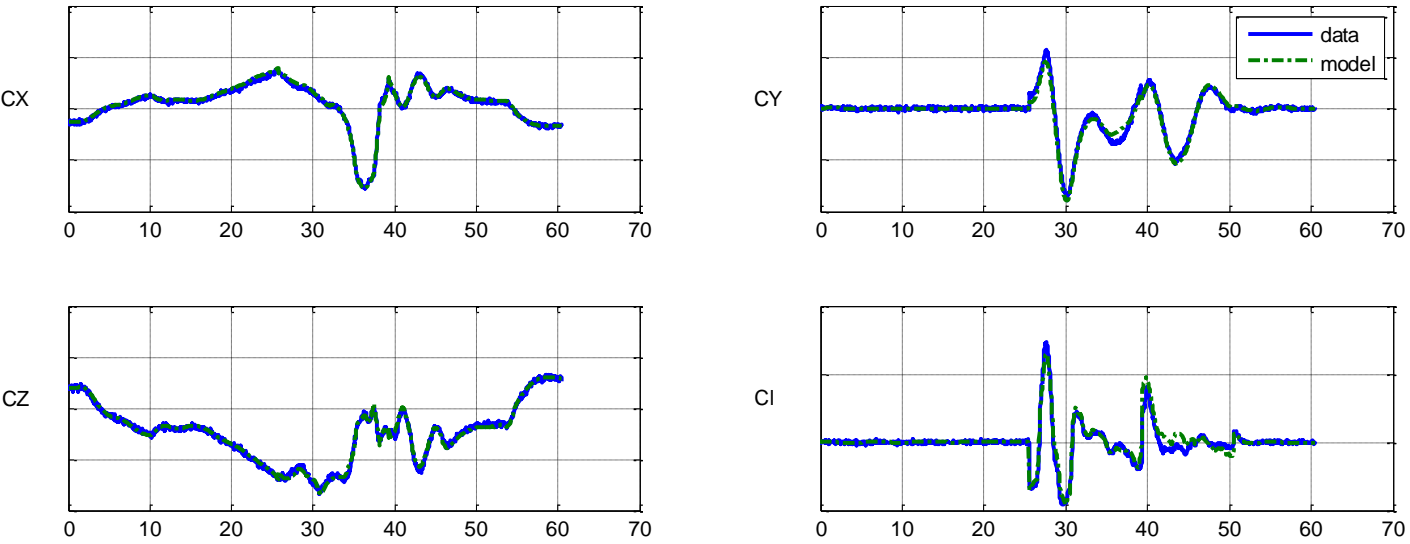

$\mathrm{Cl}$
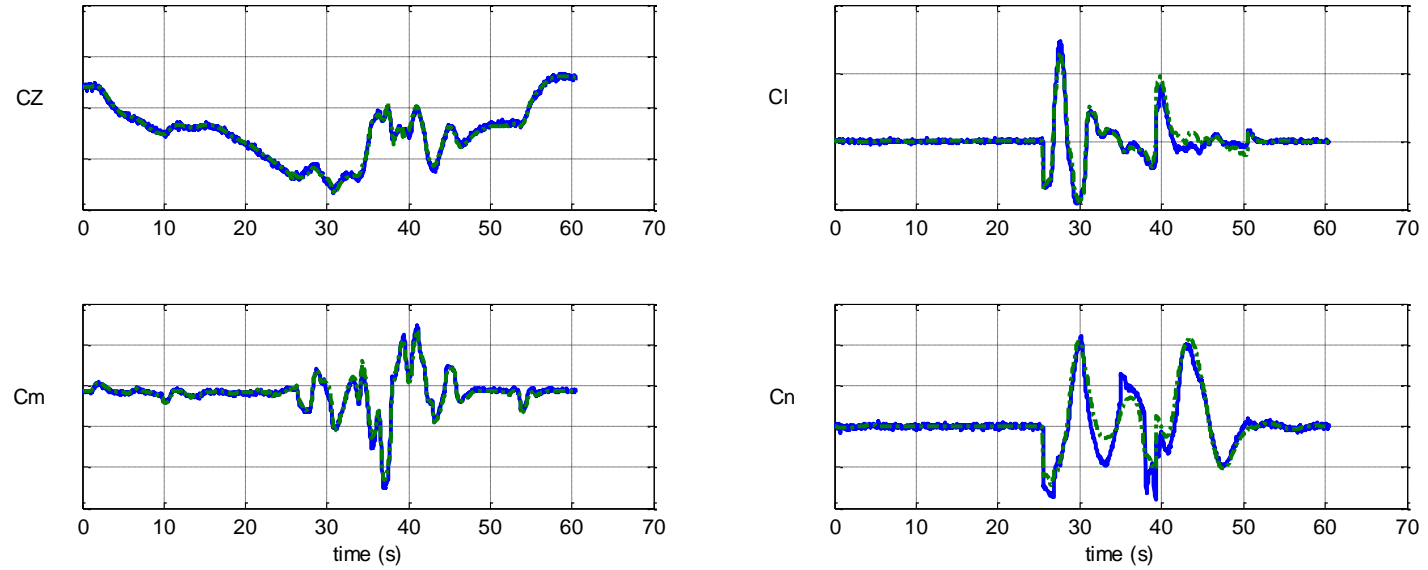

Figure 9. Stall maneuver prediction, yaw damper off

\section{Conclusions}

Simulated flight test data for a commercial jet transport aircraft was used to demonstrate techniques for efficiently and effectively identifying global aerodynamic models from piloted flight test maneuvers. The identified global aerodynamic models were shown to be accurate, with good prediction capability and easy physical interpretation. The identified models can be used for accurate flight simulation of stall, upset, and recovery, as well as normal operational flight.

A piloted flight test maneuver technique was developed and used to collect data over relatively large ranges of the explanatory variables for all six rigid-body degrees of freedom of the aircraft simultaneously. This resulted in efficient collection of highly informative dynamic flight data with de-correlated explanatory variables, for good model identifiability and modeling accuracy. The global aerodynamic modeling method incorporated spline functions in an automated orthogonal function modeling scheme, to allow accurate modeling of local complicated functional dependencies without adverse effects on other parts of the global model.

The efficiency of the approach came from the fact that the global aerodynamic modeling was automated, based on flight data alone, and also from the efficiency of the piloted flight test maneuvers. The required maneuvering is not difficult to learn or implement, and does not require flying the aircraft in unusual or dangerous flight conditions for long periods of time. The resulting global aerodynamic models exhibited good prediction capability for data from flight maneuvers that were not used in the modeling process. Global aerodynamic models identified from flight data could also serve as the basis for certifying flight simulator fidelity, e.g., using Federal Aviation Administration Level D certification standards for flight simulators. 
In addition to the intended use for accurate and efficient stall, upset, and recovery modeling, the experimentation and modeling techniques developed in this work could be used in rapid and relatively low-budget unmanned aircraft programs to save development time and money by making it possible to generate an accurate nonlinear aircraft simulation from a single flight, without the need for extensive wind tunnel testing or aerodynamic calculations. A global aerodynamic model identified from flight data alone could also be compared to aerodynamic databases generated from wind tunnel data and aerodynamic calculations to improve the fidelity of those ground-based aerodynamic prediction methods. This could be done by identifying where there are significant differences between the database generated using ground-based methods and the global model identified from flight data alone, as well as where the models agreed well. Such comparisons could also be used to estimate full-scale Reynolds number effects, or artifacts of wind tunnel testing, such as sting interference and wall effects.

The techniques described here could be applied to other commercial aircraft, such as regional jets and turboprop aircraft, and also to general aviation or military aircraft. The improved efficiency associated with this flight testing approach makes the idea of identifying an accurate flight simulation from flight data for each individual aircraft (as opposed to one simulation for all aircraft of the same make and model) a reasonable proposition. This capability could be a useful tool for improving aircraft maintenance, fault detection, envelope protection, and aviation safety.

\section{Acknowledgments}

This research in Aircraft System Identification was funded by the NASA Aviation Safety Program, Vehicle Systems Safety Technologies (VSST) project.

\section{References}

${ }^{1}$ Lambregts, A.A., Nesemeier, G., Wilborn, J.E., and Newman, R.L., “Airplane Upsets: Old Problem, New Issues”, AIAA Modeling and Simulation Technologies Conference and Exhibit, AIAA 2008-6867, Honolulu, HI, August 2008.

${ }^{2}$ Advani, S.K., Schroeder, J.A., and Burks, B., "What Really Can Be Done in Simulation to Improve Upset Training AIAA 2010-7791, AIAA Modeling and Simulation Technologies Conference, Toronto, Ontario Canada, August 2010.

${ }^{3}$ Foster, J.V., Cunningham, K., Fremaux, C.M., Shah, G.H., Stewart, E.C., Rivers, R.A., Wilborn, J.E., and Gato, W. "Dynamics Modeling and Simulation of Large Transport Airplanes in Upset Conditions," AIAA 2005-5933, AIAA Guidance, Navigation, and Control Conference and Exhibit, San Francisco, CA, August 2005.

${ }^{4}$ Shah, G.H., Cunningham, K., Foster, J.V., Fremaux, C.M., Stewart, E.C., Wilborn, J.E., Gato, W., Pratt, D.W., "WindTunnel Investigation of Commercial Transport Aircraft Aerodynamics at Extreme Flight Conditions", SAE 2002-01-2912, World Aviation Congress \& Display, Phoenix, AZ, November 2002.

${ }^{5}$ Cunningham, K., Foster, J.V., Shah, G.H., Stewart, E.C., Rivers, R.A., Wilborn, J.E., and Gato, W., "Simulation Study of a Commercial Transport Airplane During Stall and Post-Stall Flight", SAE 2004-01-3100, 2004 SAE World Aviation Congress \& Exposition, Reno, NV, November 2004.

${ }^{6}$ Cunningham, K., Foster, J.V., Shah, G.H., Stewart, E.C., Ventura, R.N., Rivers, R.A., Wilborn, J.E., and Gato, W., "Simulation Study of Flap Effects on a Commercial Transport Airplane in Upset Conditions", AIAA 2005-5908, AIAA Atmospheric Flight Mechanics Conference and Exhibit, San Francisco, CA, August 2005.

${ }^{7}$ Murch, A.M. and Foster, J.V. "Recent NASA Research on Aerodynamic Modeling of Post-Stall and Spin Dynamics of Large Transport Airplanes”, AIAA-2007-463-253, 45 th AIAA Aerospace Sciences Meeting and Exhibit, Reno, NV, January 2007.

${ }^{8}$ Shah, G.H., Foster, J.V., Cunningham, K., and Owens, D.B. "Simulation Modeling for Off-Nominal Conditions - Where Are We Today?", AIAA 2010-7792, AIAA Modeling and Simulation Technologies Conference, Toronto, Ontario Canada, August 2010.

${ }^{9}$ Cunningham, K., Foster, J.V., Morelli, E.A., and Murch, A.M., "Practical Application of a Subscale Transport Aircraft for Flight Research in Control Upset and Failure Conditions," AIAA-2008-6200, AIAA Atmospheric Flight Mechanics Conference, Honolulu, HI, August 2008.

${ }^{10}$ Morelli, E.A. "Flight Test Maneuver Design for Efficient Aerodynamic Modeling," AIAA 2011-6672, AIAA Atmospheric Flight Mechanics Conference, Portland, OR, August 2011.

${ }^{11}$ Morelli, E.A. "Efficient Global Aerodynamic Modeling from Flight Data," AIAA-2012-1050, 50 $0^{\text {th }}$ AIAA Aerospace Sciences Meeting, Nashville, TN, January 2012.

${ }^{12}$ Brandon, J.M. and Morelli, E.A. "Nonlinear Aerodynamic Modeling From Flight Data Using Advanced Piloted Maneuvers and Fuzzy Logic," NASA/TM-2012-217778, October 2012.

${ }^{13}$ Klein, V. and Morelli, E.A., Aircraft System Identification - Theory and Practice, AIAA Education Series, AIAA, Reston, VA, 2006.

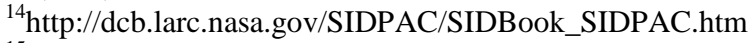

${ }^{15}$ Smith, R.M.: "A Description of the Cockpit Motion Facility and the Research Flight Deck Simulator," AIAA-2000-4174, AIAA Modeling and Simulation Technologies Conference and Exhibit, Denver, CO, August 2000.

${ }^{16}$ Chung, V. and Hutchinson, B.: "A Unique Software System for Simulation-To-Flight Research," AIAA-2001-4057, AIAA Modeling and Simulation Technologies Conference and Exhibit, Montreal, Quebec, August 2001. 
${ }^{17}$ Morelli, E.A. "Global Nonlinear Aerodynamic Modeling using Multivariate Orthogonal Functions," Journal of Aircraft, Vol. 32, No. 2, March-April 1995, pp. 270-77.

${ }^{18}$ Seher-Weiss, S. "Identification of Nonlinear Aerodynamic Derivatives using Classical and Extended Local Model Networks," Aerospace Science and Technology, Vol. 15, 2011, pp. 33-44.

${ }^{19}$ de Visser, C.C., Mulder, J.A., and Chu, Q.P. "Global Aerodynamic Modeling with Multivariate Splines," AIAA-20087500, AIAA Modeling and Simulation Technologies Conference and Exhibit, Honolulu, HI, August 2008.

${ }^{20}$ Barron, A.R., "Predicted Squared Error : A Criterion for Automatic Model Selection," Self-Organizing Methods in Modeling, Farlow, S.J., Ed., Marcel Dekker, Inc., New York, NY, 1984, pp. 87-104.

${ }^{21}$ Morelli, E.A. and DeLoach, R., "Wind Tunnel Database Development using Modern Experiment Design and Multivariate Orthogonal Functions," AIAA Paper 2003-0653, 41 $1^{\text {st }}$ AIAA Aerospace Sciences Meeting and Exhibit, Reno, NV, January 2003.

${ }^{22}$ Morelli, E.A., "Practical Aspects of the Equation-Error Method for Aircraft Parameter Estimation," AIAA-2006-6144, AIAA Atmospheric Flight Mechanics Conference, Keystone, CO, August 2006.

Table 1. Aircraft sensor random error root-mean-square values

\begin{tabular}{|c|c|}
\hline \hline$V, \mathrm{ft} / \mathrm{s}$ & 0.05 \\
\hline$\alpha, \mathrm{deg}$ & 0.025 \\
\hline$\beta, \mathrm{deg}$ & 0.025 \\
\hline$p, \mathrm{deg} / \mathrm{s}$ & 0.25 \\
\hline$q, \mathrm{deg} / \mathrm{s}$ & 0.25 \\
\hline$r, \mathrm{deg} / \mathrm{s}$ & 0.25 \\
\hline$\phi, \mathrm{deg}$ & 0.02 \\
\hline$\theta, \mathrm{deg}$ & 0.02 \\
\hline$\psi, \mathrm{deg}$ & 0.02 \\
\hline$a_{x}, \mathrm{~g}$ & 0.004 \\
\hline$a_{y}, \mathrm{~g}$ & 0.004 \\
\hline$a_{z}, \mathrm{~g}$ & 0.004 \\
\hline $\bar{q}, \mathrm{lbf} / \mathrm{ft}^{2}$ & 0.01 \\
\hline \hline
\end{tabular}

